\title{
Functional dissociation between dorsal and ventral regions of the medial prefrontal cortex in rats
}

\author{
PASCALE GISQUET-VERRIER \\ Université Paris-Sud, Orsay, France \\ GORDON WINOCUR \\ University of Toronto, Toronto, Ontario, Canada \\ and \\ BENOÎT DELATOUR \\ Université Paris-Sud, Orsay, France
}

\begin{abstract}
In order to investigate the respective roles of dorsal and ventral parts of the medial prefrontal cortex (mPFC) on a delayed alternation task, the acquisition of which is disrupted by large mPFC lesions (Winocur, 1991), rats with circumscribed lesions to the anterior cingulate (ACd) or the prelimbicinfralimbic (PL-IL) cortex were compared. As was predicted, ACd lesions severely impaired performance, and an analysis of the results suggests an involvement of this region in sequencing temporally ordered behavior. On the other hand, PL-IL lesions had no effect, a finding that was in contrast with previous evidence that PL-IL lesions induced delay-dependent deficits on a similar task (Delatour \& Gisquet-Verrier, 1999). However, an important difference between the tasks related to the scheduling of critical delays. Whereas in the previous experiment, delay intervals were increased progressively in a block design, variable intertrial intervals were a constant feature of training in the present Experiment 1. In Experiment 2, rats with PL-IL lesions were administered Delatour and Gisquet-Verrier's (1999) alternation task, but modified to incorporate Winocur's (1991) variable-interval procedure that required less response adjustment. Under these conditions, the PL-IL group performed normally, as in Experiment 1. Taken together, the results provide evidence that the PL-IL cortex is implicated in processes that support attentional mechanisms and behavioral flexibility. Overall, the present results support the general hypothesis of subregionalization of mPFC functions.
\end{abstract}

In rats, the prefrontal cortex (PFC) is divided into different regions (medial, lateral, and orbital), each possessing distinct cytoarchitectonic and connectional characteristics (Groenewegen, 1988; Krettek \& Price, 1977; Leonard, 1969; Reep, Corwin, \& King, 1996). For the most part, behavioral studies have focused on the medial part of the PFC (mPFC), largely on the basis of evidence that points to this structure as having a fundamental role in memory and related functions (see Kolb, 1984, 1990, for reviews). Much of this work has implicated the mPFC in delayed responding and in working memory (Dunnett, 1990; Harrison \& Mair, 1996; Joel, Weiner, \& Feldon, 1997; Kesner, Hunt, Williams, \& Long, 1996; Larsen \&

This research was supported by a grant to G.W. from the Natural Sciences and Engineering Research Council of Canada. B.D. was supported by a research fellowship in neurobiology from Laboratoires Lilly. We thank Paulette Richer for help in behavioral testing. Correspondence concerning this article should be addressed to P. GisquetVerrier, Laboratoire de Neurobiologie de l'Apprentissage, de la Mémoire et de la Communication (NAMC), CNRS-UMR 8620, Université Paris-Sud, Bât. 446, 91405 Orsay Cedex, France (e-mail: pascale. gisquet@ibaic.u-psud.fr).
Divac, 1978; Thomas \& Brito, 1980), but there is also considerable evidence that the structure is involved in other behavioral processes. For example, several investigators have demonstrated the adverse effects of mPFC lesions on various aspects of strategic behavior (e.g., response selection; Passingham, Myers, Rawlins, Lightfoot, \& Fearn, 1988; Winocur, 1991, 1992; Winocur \& Eskes, 1998), and there is an established literature that identifies this region with behavioral flexibility and attentional processes (Becker, Olton, Anderson, \& Breitinger, 1981; Bussey, Muir, Everitt, \& Robbins, 1997; de Bruin, 1994; Muir, Everitt, \& Robbins, 1996).

The rat $\mathrm{mPFC}$ is a heterogeneous structure that can be dissociated into dorsal and ventral parts (Condé, MaireLepoivre, Audinat, \& Crépel, 1995; Sesack, Deutsch, Roth, \& Bunney, 1989). The dorsal part includes the precentral and the anterior cingulate (ACd) cortices and maintains dense connections with sensory and motor systems, as well as projecting to the dorsal striatum (Groenewegen, Berendse, Wolters, \& Lohman, 1990; van Eden, Lamme, \& Uylings, 1992). The pattern of neural connections associated with the dorsal $\mathrm{mPFC}$ indicates that this area might be homologous to the premotor cortex of 
higher species (Preuss, 1995). On the other side, the ventral part of the MPFC, which is composed of the prelimbic (PL) and the infralimbic (IL) cortices (i.e., the PL-IL area), is involved in the limbic circuitry. The PL-IL area receives strong projections from the $\mathrm{CAl} /$ subiculum and entorhinal subfields of the hippocampal formation (Insausti, Herrero, \& Witter, 1997; Jay \& Witter, 1991), projects to the nucleus accumbens (Berendse, Galis-de Graaf, \& Groenewegen, 1992), and has reciprocal connections with the amygdala (Ray \& Price, 1992; Sesack et al., 1989).

In recent years, several investigators have examined the effects of selective lesions to the PL-IL and/or the ACd areas in a variety of behavioral paradigms. This work has given rise to various specific hypotheses regarding the functional significance of the respective regions. For example, Ragozzino, Adams, and Kesner (1998) have proposed that the ACd area is involved in working memory for egocentric space, whereas the PL-IL area is involved in working memory for allocentric space. More recently, the same authors have confirmed that these regions make different contributions to cognitive functions, with the PLIL area, but not the ACd area, being involved in switching behavioral guiding strategies (Ragozzino, Wilcox, Raso, \& Kesner, 1999). Earlier, Seamans, Floresco, and Phillips (1995) concluded that the PL cortex plays a critical role in prospective planning and that the ACd area is implicated in the flexible control of behavior that is necessary for selecting appropriate responses and altering strategies.

Work in our respective laboratories has also been concerned with this issue (e.g., Delatour \& Gisquet-Verrier, 1996, 1999, 2000; Winocur, 1991, 1992; Winocur \& Eskes, 1998). For example, Delatour and Gisquet-Verrier (1999) found that PL-IL lesions did not affect acquisition of a go/no-go conditional discrimination task but did induce a time-dependent deficit when delays were introduced between stimulus presentation and the opportunity to respond. By comparison, rats with large lesions to the $\mathrm{mPFC}$ that affected mainly dorsal regions were consistently impaired in conditional rule learning on similar tasks, even at very short delays (Winocur, 1991, 1992; Winocur \& Eskes, 1998; see also Passingham et al., 1988). The combined data led Delatour and Gisquet-Verrier (1999) to suggest, in line with the views outlined above, that the dorsal mPFC contributes to the process of response selection, whereas the ventral mPFC participates in the organization of delayed responding.

A difficulty with work in this area is that conclusions often are based on studies that vary in numerous waysmost importantly, in terms of the location and size of lesions. Accordingly, one objective of the present study was to test the above hypotheses by comparing rats with circumscribed lesions that were restricted to the PL-IL or the ACd areas on a variable-interval (VI), delayed-alternation task that was shown to be sensitive to large dorsal mPFC lesions (Winocur, 1991). In this task, conducted in an operant chamber, rats were presented with a series of al- ternating reinforced and nonreinforced trials (go/no-go schedule) with a variable $(0-80 \mathrm{sec})$ intertrial interval (ITI). Successful performance on this task requires the rat to learn the basic response alternation rule and also be able to retain trial-specific information over short and long ITIs. We predicted a functional dissociation in which the ACd group would exhibit the deficit pattern observed by Winocur (1991; i.e., impairment in conditional rule learning). In contrast, in line with a working memory hypothesis, the deficit of the PL-IL group was expected to be more selective, affecting primarily performance at longer delays.

\section{EXPERIMENT 1}

\section{Method}

\section{Subjects}

Thirty-four male Long-Evans rats ( $50-57$ days old), weighing $250-300 \mathrm{~g}$ at the start of the experiment, were obtained from the Trent University Breeding Center (Peterborough, Ontario). Rats were raised in social groups until they were 3-4 months old, at which time they were transported to individual wire-mesh cages and maintained on a 12:12-h day:night cycle with free access to food and water. Cages were located in a temperature-controlled room $\left(21^{\circ} \mathrm{C}\right)$ in which the relative humidity was maintained at about $35 \%$.

\section{Surgery}

The rats were assigned randomly to one of the three surgical groups: the PL-IL $(n=12), \mathrm{ACd}(n=11)$, and sham-operated (Sham, $n=11$ ), groups. The rats were injected with atropine (i.p. injection, $0.3 \mathrm{ml}$ ) and were anesthetized $15 \mathrm{~min}$ later with pentobarbital $(50 \mathrm{mg} / \mathrm{kg} / \mathrm{i}$.p.) before being mounted in a stereotaxic frame (Narashige Instruments, Tokyo, Model SR6). The skin on the skull was incised, and underlying tissue was removed. Horizontality of the head was verified, and holes were drilled around each injection's site. Ibotenic acid (Sigma Chemical Company, St. Louis) dissolved in phosphate buffered saline $(10 \mathrm{mg} / \mathrm{ml}$, final $\mathrm{pH}$ of 7.4$)$ was injected bilaterally through a cannula (180 $\mu \mathrm{m}$ in diameter), using a microinjector. Bilateral lesions of the $\mathrm{ACd}$ cortex were made using the following injection coordinates (Paxinos \& Watson, 1986): $\mathrm{AP},+2.5 \mathrm{~mm}$ and $+3.8 \mathrm{~mm}$ from the bregma; $\mathrm{ML}, \pm 0.5 \mathrm{~mm}$ from midline; and DV $-1.5 \mathrm{~mm}$ from the dura (each injection, $0.15 \mu \mathrm{l}$ delivered over a $1-\mathrm{min}$ period). Bilateral PL-IL cortical lesions were performed in a similar way at the following coordinates: $\mathrm{AP},+3.6 \mathrm{~mm} ; \mathrm{ML}, \pm 0.7 \mathrm{~mm}$; and $\mathrm{DV},-3.4 \mathrm{~mm}$ (each injection, $0.5 \mu \mathrm{l}$ delivered over a $4-\mathrm{min}$ period). After each injection, the cannula was left in place for $5 \mathrm{~min}$ (ACd rats) or $10 \mathrm{~min}$ (PL-IL rats). Sham-operated rats were subjected to the same surgical procedure, but without penetration of the cannula. At the end of the surgery, the incision was sutured, and sulfamides were locally applied.

\section{Histology}

At the conclusion of behavioral testing, the rats were overdosed with pentobarbital $(120 \mathrm{mg} / \mathrm{kg})$ and perfused transcardially with saline, followed by a $10 \%$ buffered formalin solution. Brains were postfixed for one night in buffered formalin. After cryoprotection by immersion in a $30 \%$ sucrose-formalin solution for $48 \mathrm{~h}$, the brains were cut on a freezing microtome into $40-\mu \mathrm{m}$ sections. Every second section was taken, mounted on a gelatin-coated slide, and stained with cresyl violet.

Evaluation of the lesion size was performed by digitizing the lesion extent with a graphic software (Canvas, Deneba). To be included in the lesioned groups, it was necessary that cortical lesions, in each hemisphere, be damaged in more than $40 \%$ of the pregen- 
ual ACd cortex (ACd group) or in more than $50 \%$ of the PL-IL area (PL-IL group), with minor, if any, encroachment on adjacent prefrontal areas.

\begin{abstract}
Apparatus
The experiment was conducted in two identical Skinner boxes, each equipped with a single retractable lever located to the right of a central feeder. Each box was contained in a soundproof chamber $(52.5 \times 52.5 \times 52.5 \mathrm{~cm})$. A $3-\mathrm{W}$ light mounted in the ceiling of the chamber provided general illumination. Throughout pretraining and in the reinforced trials of delayed alternation testing, each leverpress produced a single 45-mg Noyes food pellet. Each Skinner box was connected to its own PC, which controlled all task contingencies and recorded behavioral measures.
\end{abstract}

\section{Procedure}

After 1 week of postoperative recovery, the rats were placed on a food deprivation schedule that gradually reduced their weight to $85 \%$ of their free-feeding weight. They were maintained at this level during the course of behavioral testing. The rats first received daily 15 min pretraining sessions on a continuous reinforcement (CRF) schedule until they made a minimum of 75 leverpresses per session over 2 consecutive days. The VI delayed alternation task began on the following day. Each session consisted of 12 reinforced (go) trials alternating with 12 nonreinforced (no-go) trials. At the beginning of each session, the lever was inserted for $20 \mathrm{sec}$ in the chamber. Each leverpress during the go trials produced a single food pellet, whereas leverpresses during no-go trials were not rewarded.

Trials were separated by a variable ITI ranging from 0 to $80 \mathrm{sec}$ $(0,5,10,20,40$, or $80 \mathrm{sec})$, during which the lever was retracted. For each session, the rats were trained with variable ITIs presented according to a pseudorandom sequence, with each delay occurring twice after a go trial and twice after a no-go trial. The sequence of ITIs was varied from day to day but always began with a go trial. The rats received one session per day for 21 days.

\section{Data Analysis}

Previous work has shown that, in the VI delayed alternation task, rats pattern their responses according to the reinforcement schedule, with shorter response latencies for the go trials than for the nogo trials (see Winocur, 1991). In the present experiment, the latency to the first leverpress was recorded for each trial. At the end of each session, the mean latencies for go and no-go trials were calculated for each delay interval, as well as the differences between the nogo and the go latencies for each delay condition ( $\Delta$ latency ratio).

Statistical analyses were performed (1) on the $\Delta$ latency ratios obtained over seven blocks of three consecutive sessions and (2) on the go and no-go latencies obtained for each ITI on the seventh block of three trials.

\section{Results}

\section{Histology}

Figure 1 illustrates a schematic reconstruction of ACd lesions (right part) and of PL-IL lesions (left part) in coronal sections from a standard stereotaxic atlas (Paxinos \& Watson, 1986). In most cases, lesions were characterized by a central cavity (in the vicinity of injection sites) surrounded by nonfunctional necrotic tissues composed of glial cells and picnotic-shaped neurons. Cytoarchitectonic nomenclature for the MPFC advanced by Krettek and Price (1977) was used in the description of cortical lesions in the present study.

Dorsal anterior cingulate cortical lesions. All the rats that fulfilled the histological criteria sustained a par- tial lesion of the pregenual part of the ACd cortex. Only restricted encroachments into the dorsal PL cortex were noted and, on the dorsal extent, the medial precentral cortex was generally spared. Five rats were rejected owing to extensive invasion of the PL cortex. The remaining 6 rats sustained a restricted and subtotal $\mathrm{ACd}$ lesion (mean damage, $61.61 \% \pm 6.28 \%$; minimum, $40.36 \%$; maximum, $85.41 \%)$.

Prelimbic-infralimbic cortical lesions. The rats in this group generally presented a bilateral lesion of most of the PL-IL cortices. One rat sustained damage to the medial orbital cortex and was retained. The dorsomedial PFC cortex (ACd and medial precentral cortices) was almost always preserved in all the rats. Five rats were discarded from behavioral analyses owing to very small lesions $(n=2)$, unilateral lesions $(n=2)$, or lesions extending too far caudally and ventrally in subcallosal regions $(n=1)$. The 7 remaining rats presented bilateral lesions centered on the PL-IL area (mean damage, $66.27 \% \pm 4.24 \%$; minimum, 59.76\%; maximum, $82.32 \%$ ).

\section{Behavior}

Acquisition of the variable-interval delayed alternation task. The results obtained during training are illustrated in Figure 2 in the form of no-go-go $(\Delta)$ latency ratios. Ratios were calculated and then averaged for each of the six ITIs. A high ratio would result from shorter latencies in the go trials than in the no-go trials. Thus, improved performance on the task over test sessions corresponded to progressive increases of the $\Delta$ ratio.

An analysis of variance (ANOVA), with one betweengroup variable (lesion) and one within-group measure (block), was performed on the $\Delta$ latency ratios obtained for the three experimental groups over the seven blocks of three training sessions. This analysis yielded a significant main effect of lesion $[F(2,21)=6.10, p<.01]$, as well as a significant main effect of block $[F(6,126)=14.11$, $p<.001]$, with no lesion $\times$ block interaction $[F(12,126)=$ 1.66 , n.s.]. Complementary analyses revealed progressively improved performance for each of the three experimental groups [Sham, $F(6,60)=5.45, p<.001 ; \mathrm{PL}-\mathrm{IL}$, $F(6,36)=8.23, p<.001 ; \mathrm{ACd}, F(6,30)=4.22, p<$ $.005]$. The ACd-lesioned group, however, exhibited significantly lower $\Delta$ latency ratios than did the sham-operated group $[F(1,15)=18.30, p<.001]$ and the PL-ILlesioned group $[F(1,11)=4.53, p=.053]$, whereas the PL-IL and Sham groups did not differ from each other $(F<1)$. Paired comparisons indicated that the ACd group had significantly lower $\Delta$ latency ratios than the Sham group on Blocks 2, 3, 5, and $7(.05>p s<.01)$ and the PL-IL group on Blocks 2,3 , and $7(.05>p s<.01)$.

Analysis of the last training block. The data for the final block were analyzed separately to assess various effects after performance on the task had stabilized. The go and the no-go latencies at each ITI for the seventh block of three test sessions are illustrated in Figure 3. A threeway ANOVA (lesion $\times$ type of trial $\times$ ITI) performed on response latencies indicated a significant main effect of 
4.7
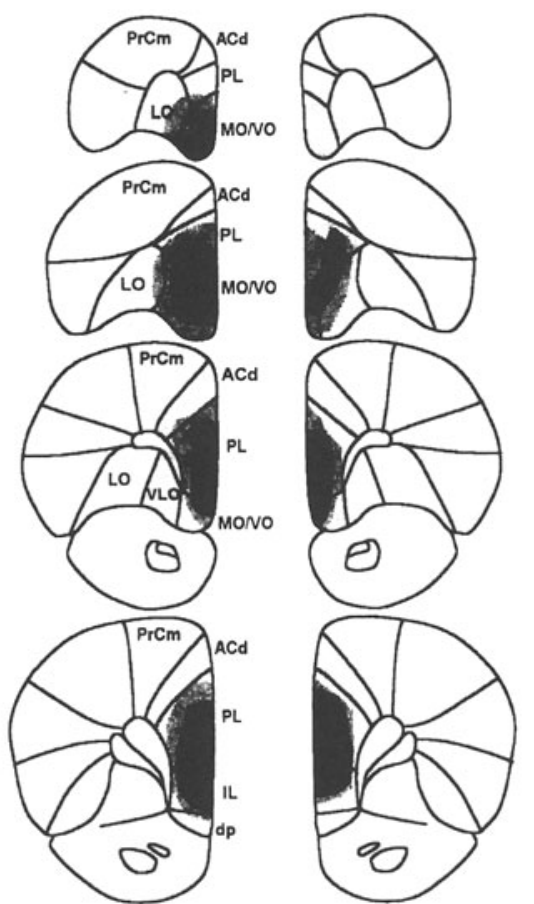

2.7
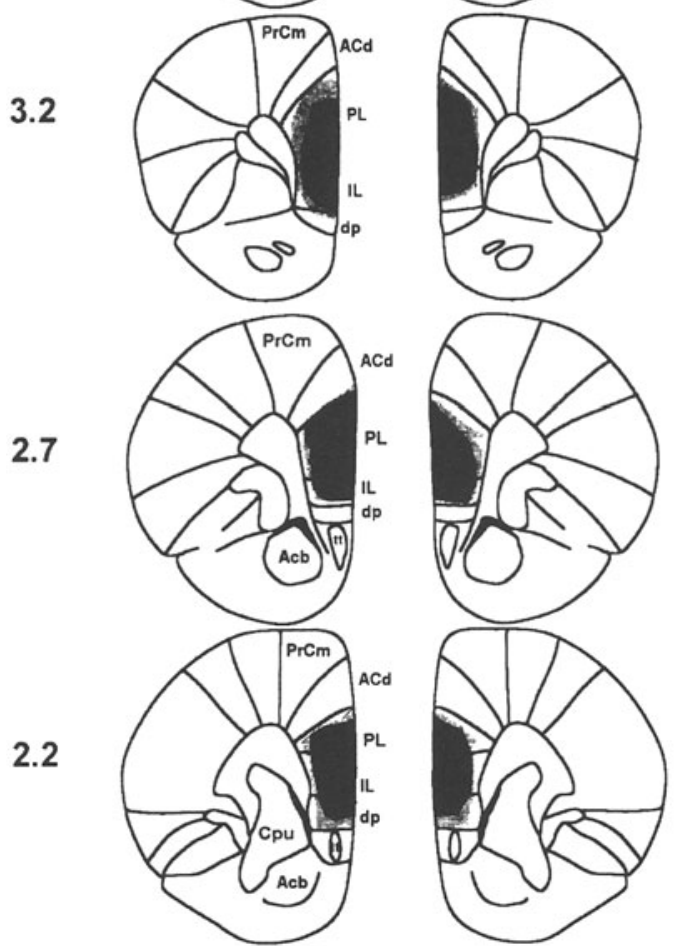

2.2
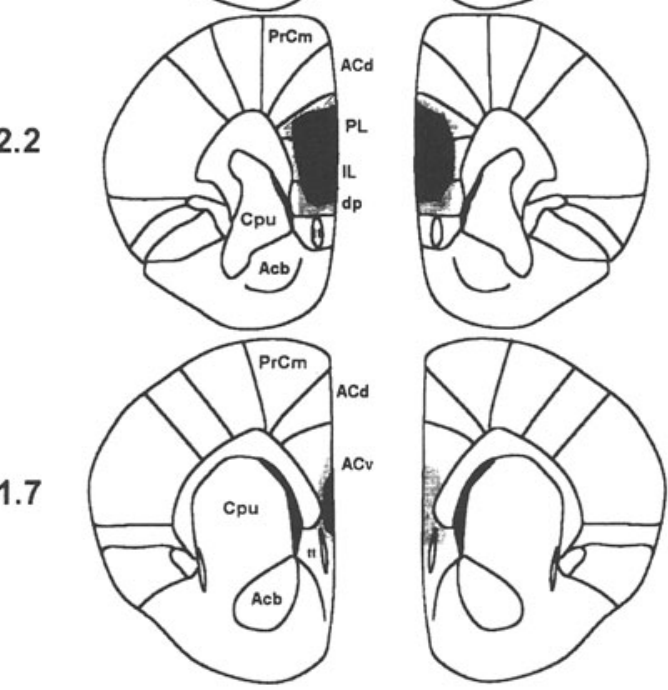

PL-IL LESIONS
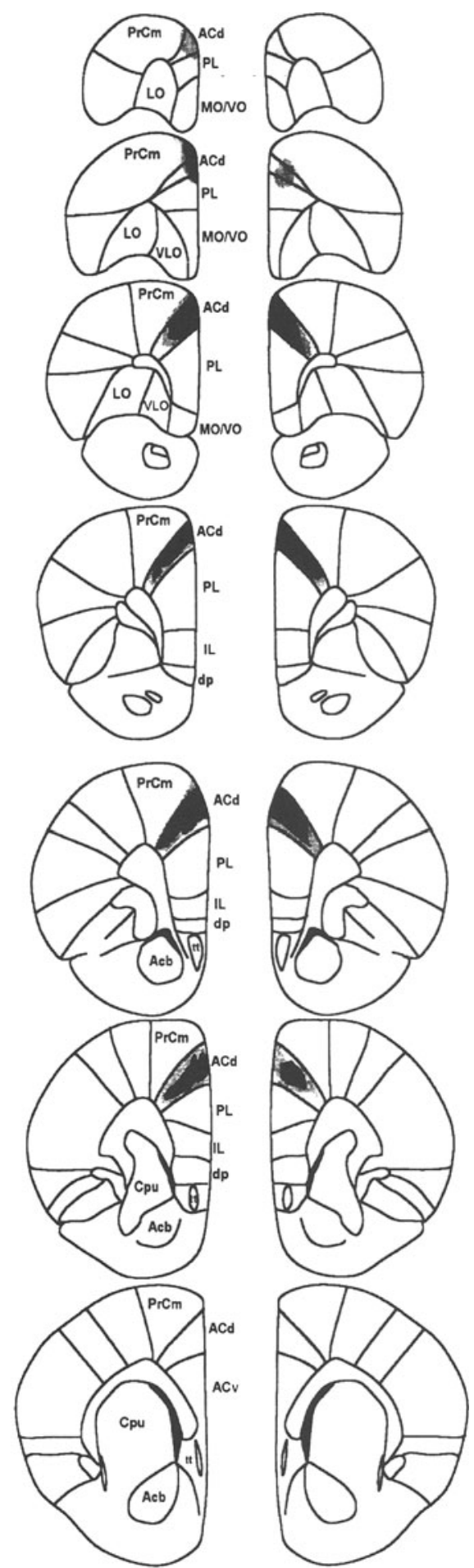

ACd LESIONS

Figure 1. Reconstruction of PL-IL (left part) and ACd (right part) cortical lesions at different rostrocaudal levels $(+4.7$ to $+1.7 \mathrm{~mm}$ from bregma) from the rats trained in Experiment 1 . Black area, region lesioned for $75 \%-100 \%$ of the rats; hatched area, region lesioned for $50 \%-75 \%$ of the rats; dotted area, region lesioned for $25 \%-50 \%$ of the rats. PL-IL lesions were centered around the ventral medial prefrontal cortex, with minor encroachments on other prefrontal cortical areas. ACd lesions were restricted to this area and did not invade adjacent PL cortex. Nomenclature is adapted from Krettek and Price (1997). PrCm, medial precentral cortex; ACd, dorsal anterior cingulte cortex; $\mathrm{ACv}$, ventral anterior cingulate cortex; $\mathrm{PL}$, prelimbic cortex; MO/VO, medial orbital and ventral orbital cortices; VLO, ventrolateral orbital cortex; LO, lateral orbital cortex; IL, infralimbic cortex; dp, dorsal peduncular cortex; tt, taenia tecta; Acb, accumbens nucleus; Cpu, caudate putamen. From The Rat Brain in Stereotaxic Coordinates (plate 5 to plate 11), by G. Paxinos and C. Watson, 1986, San Diego: Academic Press. Copyright 1986 by Academic Press. Adapted with permission. 


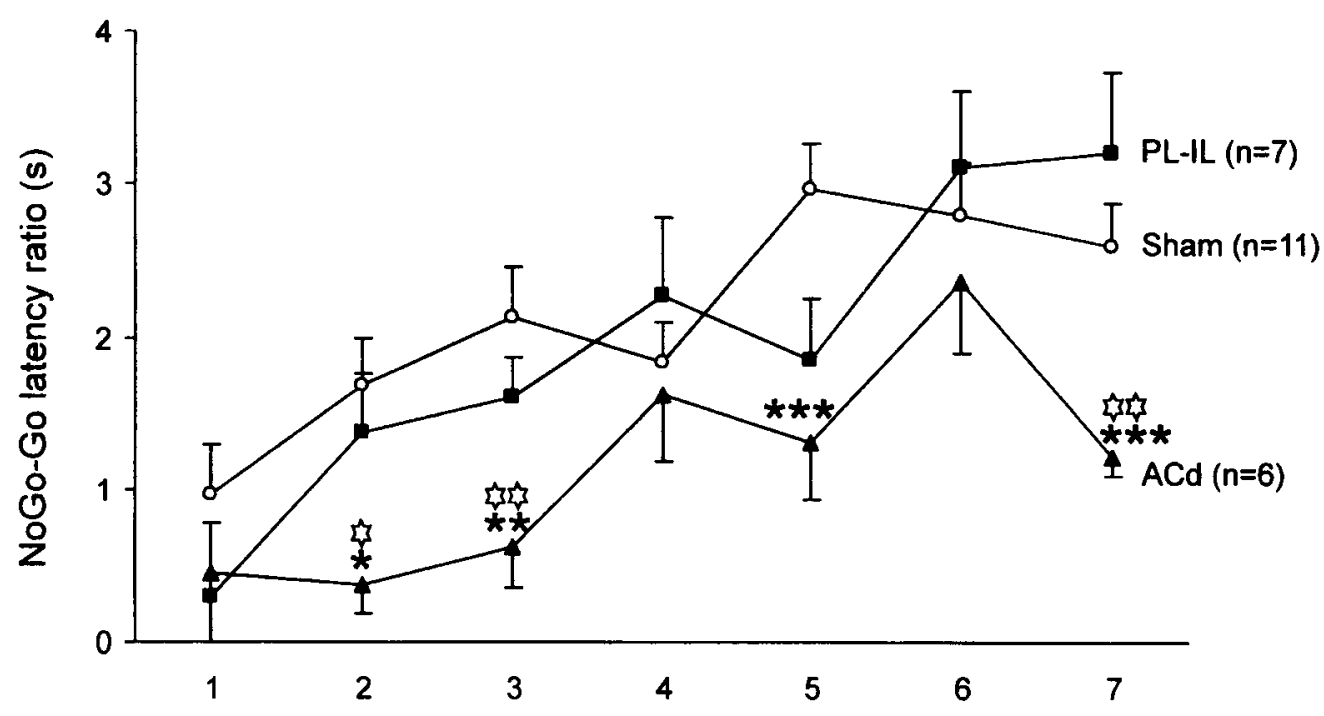

Blocks

Figure 2. Delayed go/no-go alternation task in Experiment 1. Latency ratios (no-go latencies minus go latencies in seconds; mean $\pm S E M$ ) of sham-operated rats (Sham), prelimbic-infralimbic-lesioned rats (PL-IL), and dorsal-anterior-cingulate-lesioned rats (ACd). Ratios were calculated for seven blocks of three consecutive sessions, independently of the delays. ACd rats showed a performance impairment throughout training relative to both Sham and PL-IL rats. Between group comparisons: ACd versus Sham, ${ }^{*} p<.05,{ }^{* *} p<.01,{ }^{* *} p<.001$; ACd versus $\mathrm{PL}-\mathrm{IL}$, is $p<.05$, it thp $<.01$.

lesion $[F(2,21)=4.81, p<.05]$, as well as a significant effect of type of trial $[F(1,21)=142, p<.001]$ and a significant effect of ITl $[F(5,105)=4.25, p<.002]$. There was a significant interaction between lesion $\times$ type of trial $[F(2,21)=6.71, p<.01]$, indicating that the three groups did not perform similarly during the go and the no-go trials, as well as a significant interaction between type of trial $\times \operatorname{ITI}[F(5,105)=10.16, p<.001]$, showing that the go and the no-go latencies were differentially affected by the length of the ITIs. There was no lesion $\times$ ITI interaction $(F<1)$, indicating that the length of the ITI similarly affected the three groups.

Paired comparisons showed that the ACd group significantly differed from both the Sham group $[F(1,15)=$ $4.06, p<.05]$ and the PL-IL group $[F(1,11)=10.26$, $p<.005]$, whereas there was no difference between the PL-IL group and the Sham group $[F(1,16)=2.21$, n.s.]. Complementary analyses indicated that the ACd group had significantly shorter no-go latencies than did the Sham group $[F(1,15)=6.35, p<.05]$ but these groups did not differ on the go latency measure $(F<1)$. In addition, the ACd group exhibited shorter response latencies than did the PL-IL group for both the no-go and the go latencies $[F(1,11)=11.44, p<.01$, and $F(1,11)=6.11, p<.05$, respectively].

\section{Discussion}

The results of Experiment 1 indicated that ACd lesions impaired the acquisition of a go/no-go alternation task when trained under a variable ITI procedure. This dis- ruptive effect was not modulated by the length of the ITI and seems to result from difficulties in withholding leverpressing during the no-go trials. Moreover, the effect was not due simply to a slight delay in learning the task, since it was still present at the end of testing (i.e., after 21 sessions). The behavior of those rats with ACd lesions, which was very similar to that of the rats with large dorsal mPFC lesions in Winocur's (1991) study that involved the same task, underscores the importance of this region for learning and performing the go/no-go alternation task.

In contrast, PL-IL lesions did not affect performance of the delayed alternation task at any delay. This result was surprising in that such lesions consistently produced delay-dependent deficits on similar behavioral tasks, including a conditional discrimination test involving go/nogo responding (Delatour \& Gisquet-Verrier, 1996, 1999, 2000). This finding appears to challenge the hypothesis that this region is involved in working memory.

There are at least two possible explanations for this unexpected outcome. First, there may have been some idiosyncratic elements in the present task that allowed the PL-IL rats to respond normally even at relatively long delays. It has been suggested that, in some circumstances, rats can adopt alternative strategies that may prevent a delay-dependent deficit to occur (see Chudasama \& Muir, 1997). Alternatively, the result may be due to specific training and design-related features of the present experiment. In their previous studies, Delatour and Gisquet-Verrier $(1996,1999,2000)$ used a block design in which rats were trained on a given task with minimal 

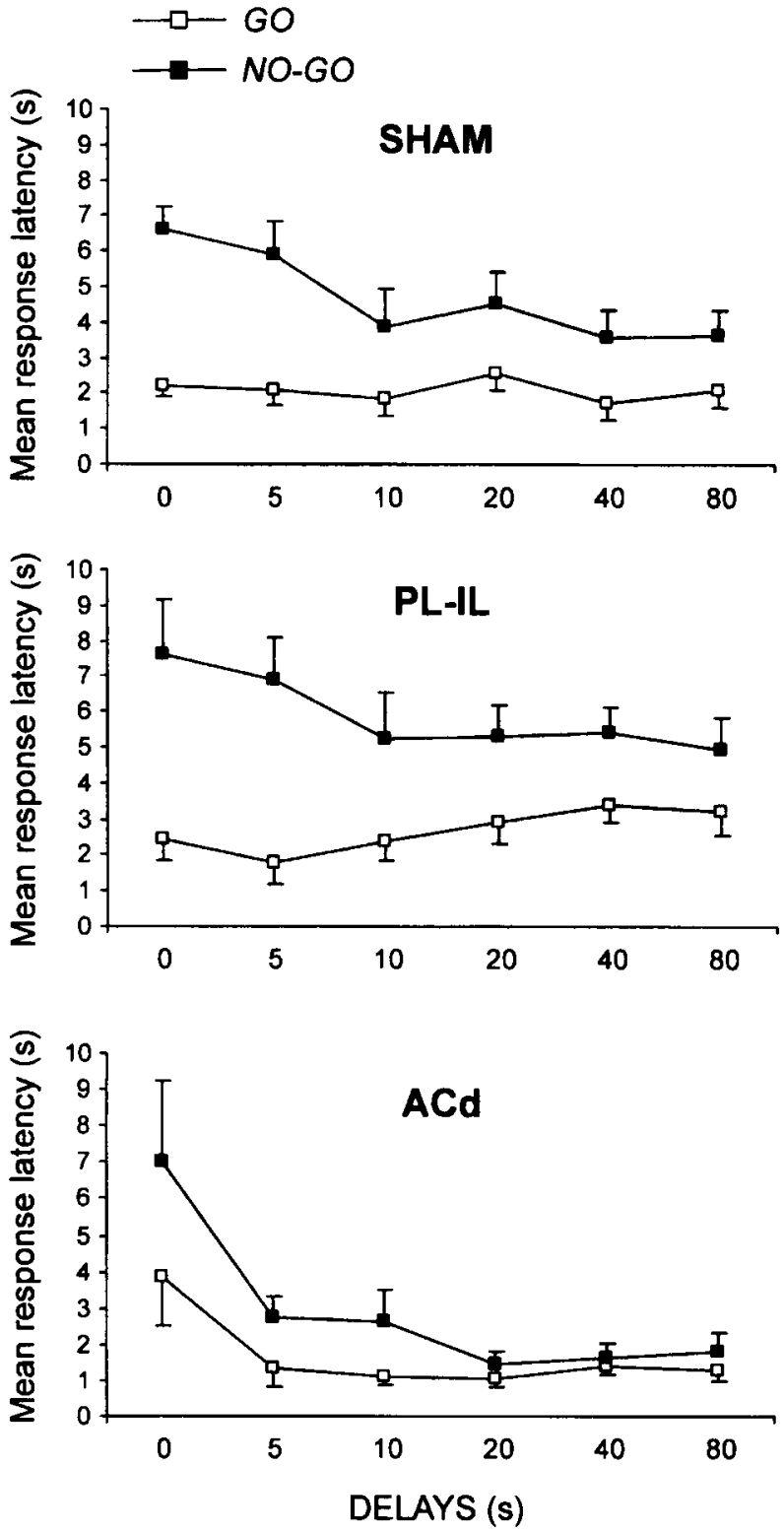

Figure 3. Delayed go/no-go alternation task in Experiment 1. Mean ( $\pm S E M$ ) response latencies (in seconds) for the go and nogo trials obtained during the last block of three training sessions (i.e., the seventh block), for sham-operated rats (Sham), prelimbic-infralimbic-lesioned rats (PL-IL), and dorsal-anteriorcingulate-lesioned rats ( $\mathrm{ACd}$ ). Response accuracy decreased with extended delays in every group of rats. Note the reduced discrimination between the go and the no-go trials in ACd rats, mainly owing to short response latencies on no-go trials.

delays. Following acquisition, rats received additional blocks of trials at increasingly longer delays. This manipulation introduced successive changes to the experimental procedure, to which rats must adjust in order to perform successfully. In Winocur's (1991) task, the variable ITI schedule was introduced at the beginning of testing and was a constant feature of the daily sessions. Thus, although rats must retain trial-specific information for vari- able delays throughout testing, this was an invariant part of the procedure that required no special adjustment.

For the following experiment, it was decided to test the effects of PL-IL lesions on a variation of the go/no-go conditional discrimination task that revealed clear delaydependent impairment when a block design was used (Delatour \& Gisquet-Verrier, 1999). The aim of Experiment 2 was to investigate whether such a delay-dependent deficit can still be evidenced when a variable-delay schedule, similar to that followed in Experiment 1, was adopted. In this test, conducted in operant chambers, rats had to learn to leverpress in response to a light stimulus in order to obtain food reward and to refrain from pressing in the presence of a tone in order to avoid shock. A variable delay $(0-20 \mathrm{sec})$ between stimulus presentation and the appearance of a retractable lever was introduced in the first session and maintained throughout testing. If the deficit observed by Delatour and Gisquet-Verrier (1999) in their progressive delay paradigm was due to an inability to adjust to increasingly demanding changes to the response requirements, it is reasonable to expect that rats with PL-IL lesions would have less difficulty with the present task, which makes fewer demands on such processes. Such an outcome would be consistent with Delatour and GisquetVerrier's (1999) suggestion that a working memory hypothesis is insufficient to account for PL-IL functions.

\section{EXPERIMENT 2}

Method
Subjects
Twenty-one male Sprague-Dawley rats were assigned to one of
two groups: sham-operated rats (Sham, $n=9$ ) and PL-IL-lesioned
(PL-IL, $n=12$ ) groups, using the same surgical and histological
procedures as those described for Experiment 1 . Housing condi-
tions were also identical to those in Experiment 1 .

\section{Apparatus}

Two operant chambers (Campden Instruments, U.K., Model 410) enclosed in sound-attenuated boxes (Model 412) were used for this experiment. Chambers were constructed of aluminum, with the exception of a Plexiglas aperture panel. The grid floor was made of 16 stainless barrels $(0.5 \mathrm{~cm}$ in diameter $)$ spaced $1 \mathrm{~cm}$ apart and was connected to a shock generator that could deliver a scrambled footshock. The left panel of each chamber was equipped with a central food magazine coupled to a 45-mg Noyes food pellets dispenser (Model 441) and a retractable lever, $10 \mathrm{~cm}$ to the right of the food tray. A $2-\mathrm{W}$ bulb was placed $10 \mathrm{~cm}$ above the food magazine, and a speaker, through which a tone $(1000 \mathrm{~Hz}, 80 \mathrm{Db})$ could be emitted, was mounted on the ceiling of the cage. Lighting of the operant chambers was provided by a $2-\mathrm{W}$ houselight mounted on the ceiling, and a masking noise (about $40 \mathrm{~dB}$ ) was provided by a ventilation fan placed on the right wall of the sound-attenuated boxes. Programming of experimental contingencies and data acquisition were accomplished by means of a PC. For each animal, data were printed and saved on magnetic disks.

\section{Procedure}

During pretraining and training, the operant chambers were continuously illuminated with the houselight.

Pretraining. The rats in each group were equally distributed among the two cages. On Day 1, the rats were placed in the chamber (with the lever retracted) for a 30 -min period, during which two 
food pellets were automatically delivered, every $60 \mathrm{sec}$, in the food magazine. On Day 2, the rats were trained on a CRF schedule. They were placed in the chamber with the lever inserted and coated with a gruel of crushed wet food pellets. During CRF, each leverpress was reinforced by two food pellets. The session ended after 50 leverpresses or after a 30 -min period, whichever came first. Those rats that failed to press 50 times in the limited period of $30 \mathrm{~min}$ were given a second CRF session at the end of the day. On Days 3-4, leverpresses were reinforced on a $20-\mathrm{sec}$ VI schedule during a $30-$ min daily session.

Training. Training in the VI delayed conditional discrimination task began on Day 5 . At the beginning of each session, the rat was placed in the chamber with the lever retracted. After a 20 -sec period, a 5-sec tone or a 5-sec light stimulus was delivered. Immediately following removal of the stimulus, a VI $(0,5,10$, or $20 \mathrm{sec})$ occurred, followed by the insertion of the lever in the chamber. A leverpress, within a 10-sec period, triggered the retraction of the lever and produced the reinforcer associated with that particular conditional stimulus. Following the light stimulus, a leverpress produced two food pellets, whereas a leverpress following the tone stimulus led to a footshock delivered through the grid floor. The next trial was then initiated $(20-n)$ sec later, where $n$ (ranging from 0 to $10 \mathrm{sec}$ ) corresponded to the response latency to press the lever during the previous trial. When no leverpress was emitted within a 10 -sec period, the lever was retracted, and the next trial was initiated $10 \mathrm{sec}$ later.

Each daily session included 56 trials for a total duration of $28 \mathrm{~min}$. Mild footshocks $(0.15 \mathrm{~mA})$ were administered during the first training session to punish responses to the tone stimulus. In subsequent sessions, the shock intensity was progressively increased until the rats consistently avoided pressing the lever in response to the tone stimulus. The rats were thus trained with different shock intensities ranging from $0.15 \mathrm{~mA}$ up to a maximal level of $0.5 \mathrm{~mA}$. The light/tone sequences were pseudorandomly determined according to one of four preplanned sequences. In every session, there were 28 light and 28 tone trials, each stimulus being delivered a maximum of three consecutive times. For each session, each of the four different delays was randomly presented seven times following tone stimuli and seven times following light stimuli.

The number of responses to the light and tone stimuli in each session was recorded. To perform successfully, the rats had to learn to press the lever following the light (go trials) and to avoid pressing the lever following the tone (no-go trials). Two types of error were defined and analyzed at the end of each session: (1) inhibition type error (pressing the lever following the tone, which led to a footshock) and (2) omission type error (omitting to press the lever following the light, which resulted in failure to obtain food reinforcement).

The rats were trained until they reached a criterion of performance for each delay condition. The criterion was defined as two consecutive training sessions in which the percentage of responses to the light was $50 \%$ higher than the percentage of responses to the tone, providing that the rats responded to the light on more than half of the light trials. The rats received at least 20 test sessions. Those that did not reach the criterion at all four delay intervals within the first 20 sessions received supplementary training until that criterion was achieved.

\section{Results}

\section{Histology}

PL-IL cortical lesions produced in the rats in Experiment 2 were very similar in terms of location and extent to those described in the first experiment. Seven rats were discarded because their lesions were considered too small. For the 5 remaining rats, lesions of the PL and the IL were extensive (global mean damage, $63.10 \% \pm 8.96 \%$; minimum, $57.78 \%$; maximum, $87.4 \%$ ) but largely spared the dorsomedial PFC. The orbitofrontal cortex was marginally damaged in 3 rats, but the encroachment was very small and not considered sufficient to justify exclusion.

\section{Behavior}

Acquisition of the variable-interval delayed conditional task. The responses made during the positively reinforced light trials (go-trials) and the negatively reinforced tone trials (no-go trials) are reported, in percentage terms, in Figure 4. Progressive differences in responding were observed between the go and the no-go trials through the successive four blocks of five training sessions. Differential responding for each type of trial was more prominent for the shortest delays than for the longest delays that separated the conditioned stimuli from the responses.

An ANOVA performed on responses to tone and light stimuli with one between-group measure (lesion) and three within-group measures (type of stimulus, delay, and block) did not reveal any effect of lesion $[F(1,12)=$ 1.47 , n.s.] but did reveal a significant main effect of block $[F(3,36)=4.37, p<.05]$, type of stimulus $[F(1,12)=$ $71.13, p<.001]$, and delay $[F(3,36)=15.33, p<.001]$.

Subsequent analysis indicated that, over the test sessions, the rats in both groups progressively increased their responses to the light stimulus $[F(3,36)=4.93, p<$ $.01]$ and concurrently reduced their responses to the tone stimulus $[F(3,36)=28.45, p<.001]$. This was confirmed by a significant block $\times$ stimulus interaction $[F(3,36)=$ $80.80, p<.001]$, which was not affected by the lesion factor $(F<1)$.

Responses to both tone and light stimuli were affected by delay $[F \mathrm{~s}(3,36)>7.84, p \mathrm{~s}<.001]$, and a significant delay $\times$ stimulus interaction $[F(3,36)=58.57, p<.001]$ indicated that the delays differentially affected responses to each stimulus. More precisely, a delay effect was observed for tone trials for all of the four training blocks $[F \mathrm{~s}(3,36)>3.05, p \mathrm{~s}<.05]$, whereas performance on light trials was affected by delay only during Blocks 2 and 3 $[F \mathrm{~s}(3,36)>3.21, p \mathrm{~s}<.05]$, suggesting a stronger effect of the delays on responses to tone than on responses to light.

Further analyses showed a significant lesion $\times$ delay interaction $[F(3,36)=3.25, p<.05]$, suggesting that the two groups differed in their sensitivity to the delays. This interaction resulted from the PL-IL rats' exhibiting a significant delay-dependent effect between the second and fourth testing blocks $[F \mathrm{~s}(3,12)>3.5, p \mathrm{~s}<.05]$, whereas Sham rats demonstrated a delay-dependent effect only during the last two conditioning blocks $\left[F_{\mathrm{s}}(3,24)>4.66\right.$, $p<.05]$.

Overall, PL-IL rats were able to perform the task adequately at all the delays. Between-group analyses performed at each ITI did not reveal any effect of lesion $[F \mathrm{~s}(1,12)<2.87$, n.s.]. Furthermore, the lesion $\times$ stimulus and the lesion $\times$ block interactions were not signifi- 

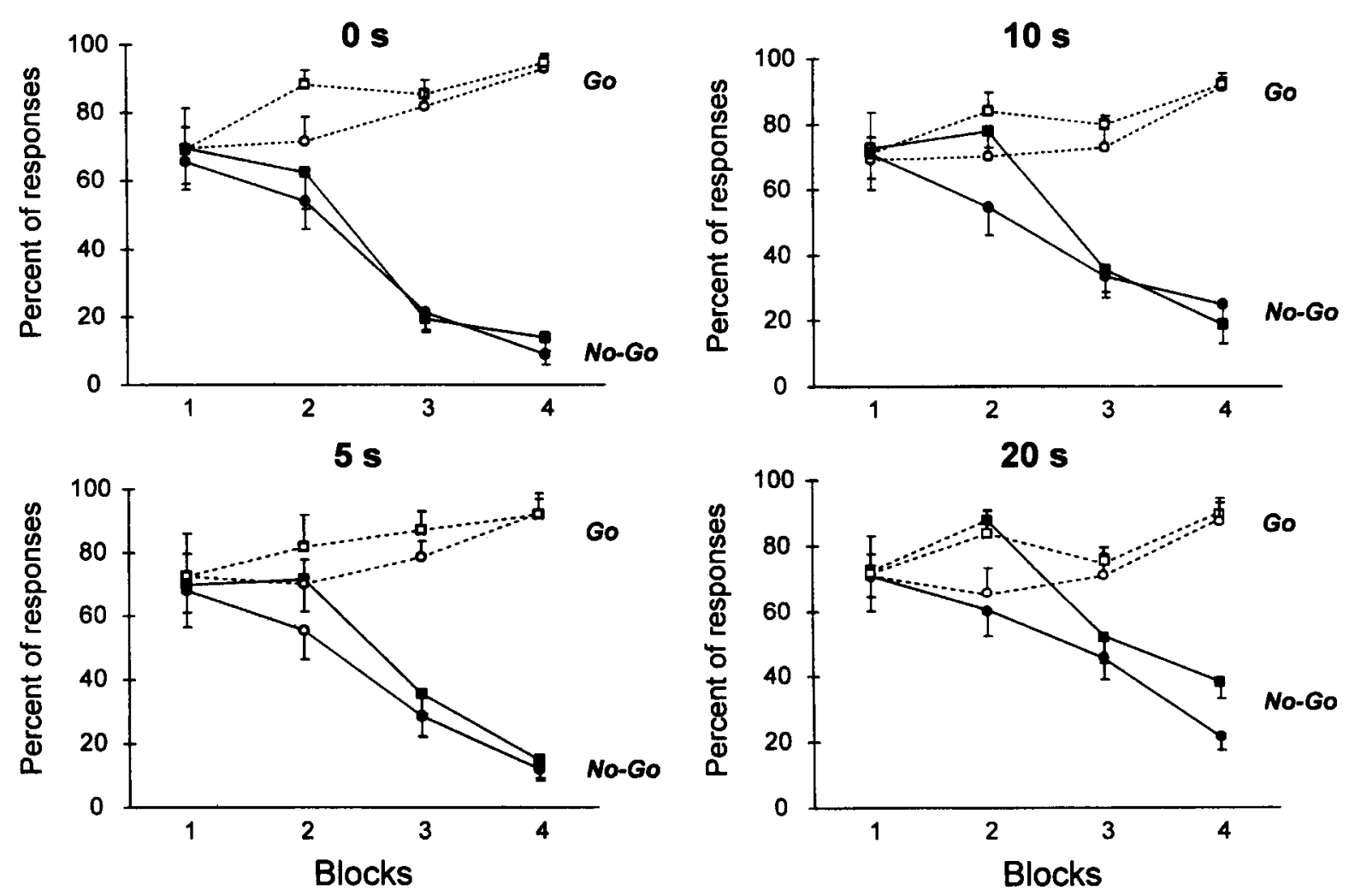

Figure 4. Delayed go/no-go conditional task in Experiment 2. Mean percentages of responses $( \pm S E M)$ of sham-operated rats (Sham, $n=9$ ) and prelimbic-infralimbic-lesioned rats (PL-IL, $n=5$ ), during the go trials (light $\rightarrow$ food pellet: Sham, -O-; PL-IL, $-\square-$ ) and the no-go trials (Tone $\rightarrow$ electrical footshock: Sham, - -; PL-IL, - $\square$-). Differences between the percentages of responses to the light and to the tone increased during the four blocks of five training sessions in every delay condition $(0,5,10$, and $20 \mathrm{sec})$. Response accuracy diminished when delays between the conditional stimulus and the response increased, with no difference between the Sham and the PL-IL groups.

cant $(F \mathrm{~s}<1)$, indicating that the lesion did not affect performance on the conditional task, regardless of the length of the delay.

Errors to criterion. Analyses were performed on the number of each type of error (inhibition error, corresponding to a leverpress following the tone stimulus; omission error, corresponding to failure to leverpress following the light stimulus) made by the rats before reaching the criterion of performance at each ITI (not illustrated). A three-way ANOVA (lesion $X$ type of errors $X$ delay) performed on the total number of errors revealed no effect of lesion $(F<1)$. However, there was a significant effect of type of error $[F(1,12)=24.9, p<.001]$, indicating that the rats made significantly more inhibition errors than omission errors, as well as a significant effect of delay $[F(3,36)=9.8, p<.001]$, indicating better performance at the shorter ITIs than at the longer ones. None of the interactions between these three factors was significant $(F \mathrm{~s}<1)$. Complementary analyses indicated a significant effect of the delay on the inhibition errors $[F(3,36)=34.38, p<.001]$, but not on the omission errors $[F(3,36)=2.45, p<.07]$.
Sessions to criterion. The number of training sessions necessary to reach the criterion at each ITI is illustrated in Figure 5. One rat in each group failed to reach all the criteria within 20 testing sessions. An ANOVA performed on these data did not reveal an effect of lesion $(F<1)$ but did indicate a significant effect of delay $[F(3,36)=$ $14.61, p<.001$ ], with more extensive training required for the longer ITIs. In addition, there was no lesion $x$ delay interaction $(F<1)$, indicating that the lesions did not modulate the general effect of the delays.

\section{GENERAL DISCUSSION}

The present study confirmed that mPFC subregions in rats differentially contribute to cognitive function (Joel, Tarrasch, Feldon, \& Weiner, 1997; Joel, Weiner, \& Feldon, 1997; Kesner et al., 1996; Ragozzino et al., 1998; Ragozzino, Wilcox, et al., 1999; Seamans et al., 1995). In Experiment 1, lesions to the ACd cortex severely disrupted go/no-go response alternation performance, thereby replicating the impairment observed by Winocur (1991) in rats with more extensive lesions to the same area. In 


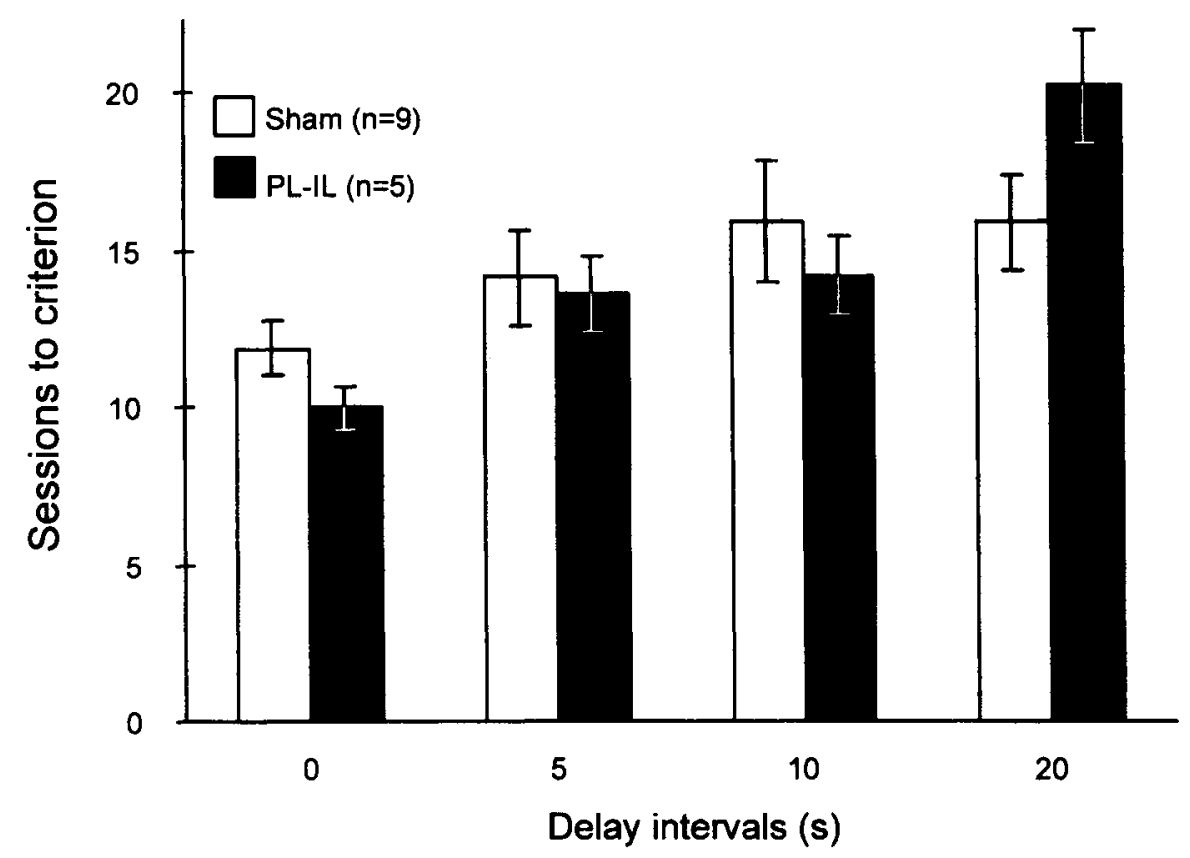

\begin{abstract}
Figure 5. Delayed go/no-go conditional task in Experiment 2. Mean number of training sessions $( \pm S E M)$ necessary to reach training criterions for each delay for sham-operated rats (Sham) and prelimbic-infralimbic-lesioned rats (PL-IL). The number of sessions increased with the length of the delay interposed between the conditional stimulus and the response, with no effect of the lesion.
\end{abstract}

contrast, lesions of the PL-IL area did not lead to a performance deficit on this task. This pattern of results was only partially expected. Although we predicted an impairment in response alternation rule learning in the ACd group, we also predicted, on the basis of previous evidence, performance deficits at relatively long delays in rats with PL-IL lesions (Brito \& Brito, 1990; Delatour \& Gisquet-Verrier, 1996, 1999, 2000).

To assess whether this discrepancy in results was due to different demands on the scheduling of responses, in Experiment 2, rats with PL-IL lesions were administered Delatour and Gisquet-Verrier's (1999) conditional discrimination task, but with a significant change. In the original task, in which rats with PL-IL lesions were impaired at long ITIs, a block design was used in which ITIs were increased progressively over test sessions. In Experiment 2 , the number and duration of ITIs were invariant throughout testing, as in Experiment 1 (see Winocur, 1991). Under these conditions, there was no difference between PL-IL and control groups. The finding that the same task may or may not be sensitive to PL-IL lesions, depending on the experimental procedure used (variable delays vs. block design), forces a reconsideration of the functional role of the PL-IL area. The implications of these results for understanding the respective roles of the dorsal and ventral mPFC regions are discussed in the following sections.

\section{Functional Role for the}

\section{Dorsal Anterior Cingulate Region}

As was noted by Winocur (1991), the impairment of ACd rats in the VI delayed alternation task reflects a deficit in learning the alternation rule that is independent of the ITI. It is important to emphasize that ACd lesions did not obliterate performance on this task, since all the rats exhibited significant improvement throughout training. Nevertheless, relative to PL-IL and control rats, the ACd group exhibited widespread impairment that was reflected primarily in a failure to modulate their response latencies according to the reinforcement schedule. This effect was particularly clear for the no-go trials, where the response latencies of ACd rats were substantially shorter than those of the other two groups.

There has been relatively little investigation of the behavioral consequences of lesions restricted to the $\mathrm{ACd}$ region. The few available studies report little or no effect of these lesions on acquisition processes. For example, ACd lesions did not affect performance on tasks in which rats were required to discriminate arms of a maze on the basis of spatial or visual cues (Ragozzino et al., 1998; Ragozzino, Wilcox, et al., 1999). Rats with ACd lesions were found to be slightly or not impaired on various spatial learning and memory tasks (e.g., Joel, Tarrasch, et al., 1997; Joel, Weiner, \& Feldon, 1997; Sánchez-Santed, de Bruin, Heinsbroek, \& Verwer, 1997; Seamans et al., 1995), 
but interestingly, when obtained, these effects were always independent of working memory load. These observations are consistent with the results of Experiment 1, in which the effects of ACd lesions did not interact with the effects of delay, as would have been expected if working memory was a factor in this group's deficit.

The main difficulties resulting from ACd lesions in Experiment 1 of the present research were associated with an inability to withhold leverpressing during the no-go trials. This pattern suggests a deficit in inhibitory processes, resulting in an uncontrolled tendency to emit a welllearned, reinforced response. Such a hypothesis would account for the perseverative tendencies that have been observed in ACd-lesioned rats in other tasks (SànchezSanted et al., 1997; Seamans et al., 1995). However, arguing against this view is the finding that ACd-lesioned rats were able to withhold inappropriate responding in a go/no-go spatial recognition test and in a simple spatial discrimination task (Ragozzino et al., 1998).

Another hypothesis that would account for the learning impairment of the alternation rule in Experiment 1 is that ACd lesions interfered with response selection. A deficit in response selection has been associated with large dorsal mPFC lesions involving both the $\mathrm{ACd}$ and the $\mathrm{PrCm}$ cortices, in various conditional learning tasks (Passingham et al., 1988; Winocur, 1992; Winocur \& Eskes, 1998). In these tasks, lesioned rats were consistently impaired in selecting correct responses from various alternatives. However, when lesions are restricted to the ACd, deficits in response selection are not reliably obtained. In Experiment 1 of the present research, response selection deficits were observed only in the no-go trials, whereas a hypothesis that emphasizes this type of deficit would have predicted poor response selection on go and no-go trials. Furthermore, ACd lesions did not impair performance when rats were required to switch from a spatial to a visual-cued version of a discrimination task, suggesting that these lesions do not interfere with response selection strategies (Ragozzino, Wilcox, et al., 1999). Finally, we recently observed that rats with $\mathrm{ACd}$ lesions performed normally on a conditional discrimination task that required response selection on the basis of correct stimulus discrimination (Delatour \& Gisquet-Verrier, 1997; see also Jackson, Kesner, \& Amann, 1998, for similar results following dorsal mPFC lesions involving the ACd). Taken together, these results indicate that lesions restricted to the ACd preserve response selection in a diverse set of circumstances.

A comparison of tasks in which rats with ACd lesions exhibit normal response selection and those in which they do not may provide the basis of an alternate hypothesis of the functional role of this region. The above-mentioned tasks that are insensitive to the effects of such lesions provide a clear choice of response alternatives that is governed by salient discriminative stimuli (see also Joel, Tarrasch, et al., 1997; Joel, Weiner, \& Feldon, 1997). An important feature of these tasks is that they do not require temporal organization or response sequencing. By com- parison, successful performance on the go/no-go alternation task requires that rats pattern their responses according to different reinforcement contingencies that coexist with variable ITIs. To accomplish this, rats must adopt a strategy that links conditional rule learning, the processing of temporally ordered information, and response selection demands. We hypothesize that the specific role of the ACd is in the implementation of rulebased behavior that requires the integration of temporal cues and response sequencing components. This view derives support from earlier evidence that rats with dorsal mPFC lesions involving the $\mathrm{ACd}$ are impaired on various learning tasks that challenge timing ability (Chaillan, Marchetti, Delfosse, Roman, \& Soumireu-Mourat, 1997; Dietrich \& Allen, 1998; Olton, 1989), require temporal ordering of responses (Chiba, Kesner, \& Reynolds, 1994; Kesner \& Holbrook, 1987; Mogensen \& Divac, 1984; Mogensen \& Holm, 1994), or engage memory for frequency (Kesner, 1990).

\section{Functional Role for the Prelimbic-Infralimbic Area}

The results of Experiments 1 and 2 showed that PL-IL lesions did not affect rats' performance on our VI, delayed alternation, and delayed conditional discrimination learning tasks. This finding was somewhat surprising, given the severe delay-dependent deficits of rats with PL-IL lesions on a variant of the conditional discrimination learning task (Delatour \& Gisquet-Verrier, 1999). However, there was a major difference between the tasks, in that the latter involved the progressive introduction of delays between stimulus presentation and the opportunity to respond, whereas, in the former, the same critical delays were always present throughout testing. Thus, it would appear that time-dependent deficits following PL-IL lesions are related to the way that delays are introduced during testing.

It is clear from the results that PL-IL lesions did not affect the ability to retain specific information. Rather, they interfered with the capacity to react adequately to a longer delay that represents a change in the experimental situation, requiring behavioral adjustment. This contention is supported by previous results (Delatour \& Gisquet-Verrier, 1999) showing that extending the delays, in a block design, constantly increased the number of errors made by rats with PL-IL lesions. The same effect was observed to a lesser degree in control rats. In Experiment 2 of the present research, the control group received 56 trials/sessions on the variable-delay procedure and reached criterion in about 16 sessions. By comparison, Delatour and Gisquet-Verrier (1999) administered 70 trials/session in the progressive-delay procedure, and control rats required 22 trials to learn the task.

Thus, a progressive-delay procedure increases task difficulty and appears to force a reengagement of learning processes that facilitates behavioral adjustment to the new task contingencies. The importance of this variable with respect to PL-IL function is highlighted by 
other evidence that delay-dependent deficits following lesions to this area are consistently associated with the progressive-delay procedure (Brito \& Brito, 1990; Delatour \& Gisquet-Verrier, 1996, 1999, 2000). In contrast, such deficits have not been reported in tasks that employed a variable-delay procedure (Aggleton, Neave, Nagle, \& Sahgal, 1995; Chudasama \& Muir, 1997; Ragozzino et al., 1998).

The present results showing no effect of PL-IL lesions even for extended delays is of particular importance because they question the hypothesis that this area is involved in working memory (Brito \& Brito, 1990; Delatour \& Gisquet-Verrier, 1996, 1999, 2000; Ragozzino et al., 1998). Kesner and his colleagues (Kesner, 2000; Ragozzino et al., 1998) take the view that involvement of the PL-IL region in working memory is restricted to spatial and visual object information. Although this hypothesis could be applied to the present results, we have already noted that a working memory hypothesis, even one that is restricted to the spatial modality, cannot fully account for the behavioral deficits resulting from damage to this area. It is well established that PL-IL lesions do not consistently affect performance in spatial working memory tasks. For example, rats with PL-IL lesions performed normally in a standard elimination task in a radial arm maze (Delatour \& Gisquet-Verrier, 1996), even when working memory was challenged by introducing delays between arm entries (Joel, Tarrasch, et al., 1997). As well, it was found that PL-IL lesions interfered with performance on spatial reference memory tasks that required a high level of behavioral flexibility but in which there were no working memory components (Delatour \& Gisquet-Verrier, 2000; see also Granon \& Poucet, 1995, for similar results).

An alternative hypothesis that takes into account the various effects of PL-IL lesions emphasizes the structure's role in processes related to attention and behavioral flexibility. PL-IL involvement in attentional mechanisms has been suggested by other investigators (Bussey et al., 1997; Granon, Hardouin, Courtier, \& Poucet, 1998; Granon, Save, \& Buhot, 1996), following observations of lesion-induced deficits in several experimental paradigms. For example, Granon and colleagues (Granon et al., 1998) showed that the PL cortex plays a central role in maintaining sustained attention that is necessary to detect subtle brightness variations. As well, there is growing evidence that the PL-IL area contributes to behavioral flexibility. For example, de Bruin, Sánchez-Santed, Heinsbroek, Donker, and Postmes (1994) reported that PL-IL lesions disrupted rats' ability to switch from a spatial to a cued version of the hidden-platform task in the Morris water maze. Other investigators have demonstrated similar switching difficulties in other paradigms in rats subjected to temporary or permanent disruption of PLIL function (Joel, Weiner, \& Feldon, 1997; Ragozzino, Detrick, \& Kesner, 1999; Ragozzino, Wilcox, et al., 1999;
Seamans et al., 1995). As well, there is evidence that PL-IL lesions do not interfere with some forms of behavioral flexibility, as is required, for example, in reversal learning. For instance, PL-IL rats were able to adapt their performance in a task involving four baited arms and four nonbaited arms in a radial maze when the two sets of arms were reversed (Joel, Tarrasch, et al., 1997) and in a water maze when the location of the hidden platform was modified (Granon \& Poucet, 1995). The lack of effect of PL-IL lesions on reversal learning may be modulated by task difficulty (Bussey et al., 1997). These investigators found that mPFC lesions (centered around the PL area) did not affect reversal learning for easily discriminable stimuli (stimuli that differed in size and brightness), but they disrupted reversal for stimuli that were more difficult to discriminate (stimuli that only differed in brightness).

The hypothesis that behavioral deficits associated with PL-IL damage can be linked to demands on cognitive processes that control adjustments to changes in task contingencies is consistent with a large body of evidence. However, the relative involvement of attentional and response control mechanisms needs to be clarified. In this regard, a recent study by Ragozzino, Detrick, and Kesner (1999) is particularly interesting. These investigators trained rats in a place or in a response-learning task in a cross-maze and then proposed either a reversal of the initial discrimination or a switch of the discrimination rule. They found that PL-IL inactivation affected behavioral flexibility only for cross-modal switching (spatial vs. cued version), but not for intramodal switching (reversal learning), supporting the view that the PL-IL area is involved in both attention and behavioral flexibility.

\section{Conclusion}

The present results strengthen the contention that the subregions of the mPFC in rats contribute differentially to behavior and, in addition, provide insight into the respective functions of the ACd and the PL-IL regions. The results, in line with those of other laboratories, support the hypotheses that the ACd region is involved in sequencing of temporally ordered behavior, whereas the PL-IL area is critically involved in processes related to attentional mechanisms and behavioral flexibility.

\section{REFERENCES}

Aggleton, J. P., Neave, N., Nagle, S., \& Sahgal, A. (1995). A comparison of the effects of medial prefrontal, cingulate cortex, and cingulum bundle lesions on tests of spatial memory: Evidence of a double dissociation between frontal and cingulum bundle contributions. Journal of Neuroscience, 15, 7270-7281.

Becker, J. T., Olton, D. S., Anderson, C. A., \& Breitinger, E. R. P. (1981). Cognitive mapping in rats: The role of the hippocampal and frontal systems in retention and reversal. Behavioural Brain Research, 3, 1-22.

Berendse, H. W., Galis-de Graaf, Y., \& Groenewegen, H. J. (1992). Topographical organization and relationship with ventral striatal compartments of prefrontal corticostriatal projections in the rat. Journal of Comparative Neurology, 316, 314-347. 
BRito, G. N. O., \& BRITo, L. S. O. (1990). Septohippocampal system and the prelimbic sector of frontal cortex: A neuropsychological battery analysis in the rat. Behavioural Brain Research, 36, 127-146.

Bussey, T. J., Muir, J. L., Everitt, B. J., \& Robins, T. W. (1997). Triple dissociation of anterior cingulate, posterior cingulate, and medial prefrontal cortices on visual discrimination tasks using a touchscreen testing procedure for the rat. Behavioral Neuroscience, 111 , 920-936.

Chaillan, F. A., Marchetti, E., Delfosse, F., Roman, F. S., \& Soumireu-Mourat, B. (1997). Opposite effects depending on learning and memory demands in dorsomedial prefrontal cortex lesioned rats performing an olfactory task. Behavioural Brain Research, 82, 203-212.

Chiba, A. A., Kesner, R. P., \& Reynolds, A. M. (1994). Memory for spatial location as a function of temporal lag in rats: Role of the hippocampus and medial prefrontal cortex. Behavioral \& Neural Biology, 61, 123-131.

Chudasama, Y., \& Muir, J. L. (1997). A behavioural analysis of the delayed non-matching to position task: The effects of scopolamine, lesions of the fornix and of the prelimbic region on mediating behaviours by rats. Psychopharmacology, 134, 73-82.

Condé, F., Maire-Lepoivre, E., Audinat, E., \& Crépel, F. (1995). Afferent connections to the medial prefrontal cortex of the rat: II. Cortical and sub-cortical afferents. Journal of Comparative Neurology, 352, 567-593.

DE BRUIN, J. P. C. (1994). Evolution of prefrontal cortex: Comparative aspects of its behavioral functions. In R. J. Greenspan \& C. P. Kyriacou (Eds.), Flexibility and constraints in behavioral systems (pp. 185192). New York: Wiley.

de Bruin, J. P. C., Sánchez-Santed, F., Heinsbroek, R. P. W., DONKer, A., \& Postmes, P. (1994). A behavioural analysis of rats with damage to the medial prefrontal cortex using the Morris water maze: Evidence for behavioral flexibility, but not for impaired spatial navigation. Brain Research, 652, 323-333.

Delatour, B., \& Gisquet-Verrier, P. (1996). Prelimbic cortex specific lesions disrupt delayed-variable response tasks in the rat. Behavioral Neuroscience, 110, 1282-1298.

Delatour, B., \& Gisquet-Verrier, P. (1997), Functional dissociations between prelimbic and anterior cingulate parts of the medial prefrontal cortex in the rat. Society for Neuroscience Abstracts, 23, 1607.

Delatour, B., \& Gisquet-VerRIER, P. (1999). Lesions of the prelimbic and infralimbic cortices in rats do no disrupt response selection but induce delay-dependent deficits: Evidence for a role in working memory? Behavioral Neuroscience, 113, 941-955.

Delatour, B., \& Gisquet-Verrier, P. (2000). Functional role of the prelimbic-infralimbic cortices in spatial memory: Evidence for its involvement in attention and behavioural flexibility. Behavioural Brain Research, 109, 113-128.

Dietrich, A., \& Allen, J. D. (1998). Functional dissociation of the prefrontal cortex and the hippocampus in timing behavior. Behavioral Neuroscience, 112, 1043-1047.

DUNNETT, S. B. (1990). Role of the prefrontal cortex and striatal output systems in short-term memory deficits associated with ageing, basal forebrain lesions, and cholinergic-rich grafts. Canadian Journal of Psychology, 44, 210-232.

Granon, S., Hardouin, J., Courtier, A., \& Poucet, B. (1998). Evidence for the involvement of the rat prefrontal cortex in sustained attention. Quarterly Journal of Experimental Psychology, 51 B, 219-233.

Granon, S., \& Poucet, B. (1995). Medial prefrontal cortex lesions in the rat and spatial navigation: Evidence for impaired planning. $B e$ havioral Neuroscience, 109, 474-484

Granon, S., Save, E., \& Buhot, M.-C. (1996). Effortful information processing in a spontaneous spatial situation by rats with medial prefrontal lesions. Behavioural Brain Research, 78, 147-154.

GroENEWEGEN, H. J. (1988). Organization of the afferent connections of the mediodorsal thalamic nucleus in the rat, related to the mediodorsal-prefrontal topography. Neuroscience, 24, 379-431.

Groenewegen, H. J., Berendse, H. W., Wolters, J. G., \& Lohman, A. H. M. (1990). The anatomical relationship of the prefrontal cortex with the striatopallidal system, the thalamus and the amygdala:
Evidence for parallel organization. Progress in Brain Research, 85, 95-118.

HARRISON, L. M., \& MaIR, R. G. (1996). A comparison of the effects of frontal cortical and thalamic lesions on measures of spatial learning and memory in the rat. Behavioural Brain Research, 75, 195-206.

InSAusti, R., Herrero, M. T., \& WitTER, M. P. (1997). Entorhinal cortex of the rat: Cytoarchitectonic subdivisions and the origin and distribution of cortical efferents. Hippocampus, 7, 146-183.

Jackson, P. A., Kesner, R. P., \& AMANN, K. (1998). Memory for duration: Role of hippocampus and medial prefrontal cortex. Neurobiology of Learning \& Memory, 70, 328-348.

JAY, T. M., \& WITTER, M. P. (1991). Distribution of hippocampal CA1 and subicular efferents in the prefrontal cortex of the rat studied by means of anterograde transport of Phaseolus vulgaris leucoagglutinin. Journal of Comparative Neurology, 313, 574-586.

Joel, D., Tarrasch, R., Feldon, J., \& Weiner, I. (1997). Effects of electrolytic lesions of the medial prefrontal cortex or its subfields on 4-arm baited, 8-arm radial maze, two-way active avoidance and conditioned fear tasks in the rat. Brain Research, 765, 37-50.

Joel, D., Weiner, I., \& Feldon, J. (1997). Electrolytic lesions of the medial prefrontal cortex in rats disrupt performance on an analog of the Wisconsin Card Sorting Test, but do not disrupt latent inhibition: Implications for animal models of schizophrenia. Behavioural Brain Research, 85, 187-201.

KESNER, R. P. (1990). Memory for frequency in rats: Role of the hippocampus and medial prefrontal cortex. Behavioral \& Neural Biology, 53, 402-410.

KESNER, R. P. (2000). Subregional analysis of mnemonic functions of the prefrontal cortex in the rat. Psychobiology, 28, 219-228.

KESNER, R. P., \& HolbroOK, T. (1987). Dissociation of item and order spatial memory in rats following medial prefrontal cortex lesions. Neuropsychologia, 25, 653-664.

Kesner, R. P., Hunt, M. E., Williams, J. M., \& Long, J. M. (1996). Prefrontal cortex and working memory for spatial response, spatial location, and visual object information in the rat. Cerebral Cortex, 6 , 311-318.

KOLB, B. (1984). Functions of the frontal cortex of the rat: A comparative review. Brain Research Review, 8, 65-98.

KoLB, B. (1990). Animal models for human PFC-related disorders. Progress in Brain Research, 85, 501-519.

KretTek, J. E., \& Price, J. L. (1977). The cortical projections of the mediodorsal nucleus and adjacent thalamic nuclei in the rat. Journal of Comparative Neurology, 171, 157-192.

LaRSEN, J. K., \& Divac, I. (1978). Selective ablations within the prefrontal cortex of the rat and performance of delayed alternation. Physiological Psychology, 6, 15-17.

LEONARD, C. M. (1969). The prefrontal cortex of the rat: I. Cortical projection of the mediodorsal nucleus: II. Efferent connections. Brain Research, 12, 321-343.

Mogensen, J., \& Divac, I. (1984). Sequential behavior after modified prefrontal lesions in the rat. Physiological Psychology, 12, 41-44.

MOGENSEN, J., \& Holm, S. (1994). The prefrontal cortex and variants of sequential behaviour: Indications of functional differentiation between subdivisions of the rat's prefrontal cortex. Behavioural Brain Research, 63, 89-100.

MUIR, J. L., EveritT, B. J., \& RobBins, T. W. (1996). The cerebral cortex of the rat and visual attentional function: Dissociable effects of mediofrontal, cingulate, anterior dorsolateral and parietal cortex lesions on a 5-choice serial reaction time task. Cerebral Cortex, 6, 470481 .

Olton, D. S. (1989). Frontal cortex, timing and memory. Neuropsychologia, 27, 121-130.

Passingham, R. E., Myers, C., Rawlins, N., Lightfoot, V., \& FEARN, S. (1988). Premotor cortex in the rat. Behavioral Neuroscience, 102, 101-109.

Paxinos, G., \& Watson, C. (1986). The rat brain in stereotaxic coordinates. San Diego: Academic Press.

Preuss, T. M. (1995). Do rats have prefrontal cortex? The RoseWoolsey-Akert program reconsidered. Journal of Cognitive Neuroscience, 7, 1-24. 
Ragozzino, M. E., Adams, S., \& Kesner, R. P. (1998). Differential involvement of the dorsal anterior cingulate and prelimbic-infralimbic areas of the rodent prefrontal cortex in spatial working memory. $\mathrm{Be}$ havioral Neuroscience, 112, 293-303.

Ragozzino, M. E., Detrick, S., \& KeSner, R. P. (1999). Involvement of the prelimbic-infralimbic areas of the rodent prefrontal cortex in behavioral flexibility for place and response learning. Journal of Neuroscience, 19, 4585-4594.

Ragozzino, M. E., Wilcox, C., Raso, M., \& Kesner, R. P. (1999). Involvement of rodent prefrontal cortex subregions in strategy switching. Behavioral Neuroscience, 113, 32-41.

RAY, J. P., \& PrICE, J. L. (1992). The organization of the thalamocortical connections of the mediodorsal thalamic nucleus in the rat, related to ventral forebrain-prefrontal cortex topography. Journal of Comparative Neurology, 323, 167-197.

REEP, R. L., CoRwiN, J. V., \& KING, V. (1996). Neuronal connections of orbital cortex in rats: Topography of cortical and thalamic afferents. Experimental Brain Research, 111, 215-232.

Sánchez-SAnTed, F., de Bruin, J. P. C., Heinsbroek, R. P. W., \& VerWER, R. W. H. (1997). Spatial delayed alternation of rats in a T-maze: Effects of neurotoxic lesions of the medial prefrontal cortex and of Tmaze rotations. Behavioural Brain Research, 84, 73-79.

Seamans, J. K., Floresco, S. B., \& Phillips, A. G. (1995). Functional differences between the prelimbic and anterior cingulate regions of the rat prefrontal cortex. Behavioral Neuroscience, 109, 1063-1073.
Sesack, S. R., Deutsch, A. Y., Roth, R. H., \& BunNey, B. S. (1989) Topographical organization of the efferent projections of the medial prefrontal cortex in the rat: An anterograde tract-tracing study with Phaseolus vulgaris leucoagglutinin. Journal of Comparative Neurology, 290, 213-242.

Thomas, G. J., \& BRiro, G. N. O. (1980). Recovery of delayed alternation in rats after lesions in medial prefrontal cortex and septum. Journal of Comparative \& Physiological Psychology, 94, 808-818.

van Eden, C. G., Lamme, V. A. F., \& Uylings, H. B. M. (1992). Heterotopic cortical afferents to the medial prefrontal cortex in the rat: A combined retrograde and anterograde tracer study. European Journal of Neuroscience, 4, 77-97.

Winocur, G. (1991). Functional dissociation of the hippocampus and prefrontal cortex in learning and memory. Psychobiology, 19, 11-20.

Winocur, G. (1992). A comparison of normal old rats and young adult rats with lesions to the hippocampus or prefrontal cortex on a test of matching-to-sample. Neuropsychologia, 30, 769-781.

WinOCUR, G., \& EsKes, G. (1998). Prefrontal cortex and caudate nucleus in conditional associative learning: Dissociated effects of selective brain lesions in rats. Behavioral Neuroscience, 112, 89-101.

(Manuscript received February 2, 2000;

revision accepted for publication March 24, 2000.) 